(1)

CrossMark

\title{
The use of chest magnetic resonance imaging in interstitial lung disease: a systematic review
}

\author{
Chiara Romei ${ }^{1}$, Laura Turturici ${ }^{2}$, Laura Tavanti ${ }^{3}$, Jelle Miedema ${ }^{4}$, Sara Fiorini ${ }^{5}$, \\ Massimo Marletta ${ }^{5}$, Piotr Wielopolski ${ }^{6}$, Harm Tiddens ${ }^{6,7}$, Fabio Falaschi ${ }^{1}$ and \\ Pierluigi Ciet ${ }^{6,7}$
}

Affiliations: ${ }^{12}$ nd Radiology Unit, Azienda Ospedaliera Universitaria Pisana, Pisa, Italy. ${ }^{2}$ Radiology, Azienda USL Toscana nord ovest Sede di Viareggio, Viareggio, Italy. ${ }^{3}$ Dept of Surgical, Medical, Molecular Pathology and Critical Care, Azienda Ospedaliera Universitaria Pisana, Pisa, Italy. ${ }^{4}$ Dept of Respiratory Medicine, Erasmus MC, University Medical Centre Rotterdam, Rotterdam, The Netherlands. ${ }^{5} 1$ st Radiology Unit, Azienda Ospedaliero Universitaria Pisana, Pisa, Italy. ${ }^{6}$ Dept of Radiology and Nuclear Medicine, Erasmus MC, University Medical Center Rotterdam, Rotterdam, The Netherlands. ${ }^{7}$ Dept of Pediatric Pulmonology and Allergology, Erasmus MC, University Medical Center Rotterdam, Rotterdam, The Netherlands.

Correspondence: Pierluigi Ciet, Dept of Radiology and Nuclear Medicine, Erasmus MC, University Medical Center Rotterdam, P.O. Box 2060, 3000 CB Rotterdam, The Netherlands. E-mail: p.cietđerasmusmc.nl

@ERSpublications

Although MRI resolution does not yet match that of CT, MRI can play an important role for functional imaging in patients with ILD. MRI can differentiate between inflammatory and fibrotic changes for monitoring targeted therapy in patients with ILD. http://ow.ly/uicr30myy9z

Cite this article as: Romei C, Turturici L, Tavanti L, et al. The use of chest magnetic resonance imaging in interstitial lung disease: a systematic review. Eur Respir Rev 2018; 27: 180062 [https://doi.org/10.1183/ 16000617.0062-2018].

ABSTRACT Thin-slices multi-detector computed tomography (MDCT) plays a key role in the differential diagnosis of interstitial lung disease (ILD). However, thin-slices MDCT has a limited ability to detect active inflammation, which is an important target of newly developed ILD drug therapy. Magnetic resonance imaging (MRI), thanks to its multi-parameter capability, provides better tissue characterisation than thin-slices MDCT.

Our aim was to summarise the current status of MRI applications in ILD and to propose an ILD-MRI protocol. A systematic literature search was conducted for relevant studies on chest MRI in patients with ILD.

We retrieved 1246 papers of which 55 original papers were selected for the review. We identified 24 studies comparing image quality of thin-slices MDCT and MRI using several MRI sequences. These studies described new MRI sequences to assess ILD parenchymal abnormalities, such as honeycombing, reticulation and ground-glass opacity. Thin-slices MDCT remains superior to MRI for morphological imaging. However, recent studies with ultra-short echo-time MRI showed image quality comparable to thin-slices MDCT. Several studies demonstrated the added value of chest MRI by using functional imaging, especially to detect and quantify inflammatory changes.

We concluded that chest MRI could play a role in ILD patients to differentiate inflammatory and fibrotic changes and to assess efficacy of new ILD drugs.

\section{This article has supplementary material available from err.ersjournals.com}

Provenance: Submitted article, peer reviewed.

Received: July 212018 | Accepted after revision: Oct 232018

Copyright OERS 2018. ERR articles are open access and distributed under the terms of the Creative Commons Attribution Non-Commercial Licence 4.0. 


\section{Introduction}

Interstitial lung disease (ILD) includes a large group of diffuse parenchymal lung diseases that are grouped together because of similar clinical, pathological and radiological features [1].

The most frequent radiological patterns are usual interstitial pneumonia (UIP) and nonspecific interstitial pneumonia (NSIP) patterns. While in UIP the key feature is honeycombing [2], on thin-slices multi-detector computed tomography (MDCT) for NSIP the key feature is bilateral ground-glass opacity [1]. Despite thin-slices MDCT conveying the highest diagnostic confidence in ILD, the diagnosis is frequently only achieved in a multidisciplinary meeting by combining clinical, radiological and pathological information $[3,4]$. Thin-slices MDCT is especially limited on defining areas of active inflammation [5]. As a matter of fact, area of inflammation and fibrosis can have the same ground-glass opacity appearance on thin-slices MDCT [5, 6]. Hence, thin-slices MDCT cannot distinguish between pure inflammatory and fibrotic ground-glass opacity, limiting its ability to detect active inflammation areas in secondary UIP or more fibrotic NSIP patients. This differentiation is important in the treatment of ILD, because anti-inflammatory medications (i.e. corticosteroids) are currently used in ILD related to collagen vascular disease in contrary to the antifibrotics pirfenidone (Roche, Basel, Switzerland) and nintedanib (Boehringer Ingelheim, Ingelheim am Rhein, Germany), which are indicated in patients with idiopathic pulmonary fibrosis (IPF) [7, 8]. Thin-slices MDCT is also limited by poor reproducibility of visual scores for ILD abnormalities and lack of specific outcome measures to evaluate response to treatment [9]. Recently, quantitative thin-slices MDCT scoring systems have improved the sensitivity of thin-slices MDCT to monitor ILD, but they are still used in the research setting [10]. For these reasons, in clinical practice, pulmonary function tests (PFT) remain the gold standard for monitoring ILD patients, especially by using the diffusing capacity of the lung for carbon monoxide (DLCO) and forced vital capacity (FVC). However, PFT only provide a global assessment of lung function, and they cannot track regional changes of ILD [1]. Moreover, both FVC and DLCO have low sensitivity and marginal changes in the range of 5-10\% are difficult to interpret. Therefore, it is clear that there is a great need for a sensitive clinical and imaging tool to monitor ILD progression and response to treatment [11].

Magnetic resonance imaging (MRI) may overcome the limitations of computed tomography and PFT. When compared to thin-slices MDCT for image quality, chest MRI does not provide any additional information [12]. However, the main advantage of MRI is the ability to provide functional and structural information in a single examination $[13,14]$. New chest MRI techniques have recently been developed and can provide information about ventilation, inflammation, perfusion and structure which could be useful to improve assessment of ILD progression and prediction of response to ILD drug therapy [15].

To date, the limited use of MRI in ILD is related to the challenges posed by this technique, with poor signal-to-noise ratio (SNR) related to physical properties of the pulmonary parenchyma and long scan time [16]. However, recent technical advances, such as parallel imaging, multi-array phase coils and ultra-short echo-time techniques have enabled higher image quality and shorter scan time [17]. These improvements have allowed chest MRI to emerge as an alternative imaging modality for the assessment of pulmonary diseases both in children and adults [16,18]. Currently, chest MRI serves as a research tool for ILD, but it might play an important role in the near future in ILD therapeutics and monitoring. In addition to the obvious advantage of the absence of ionising radiation, chest MRI may allow higher tissue characterisation and differentiation between inflammatory and pure fibrotic ground-glass opacity $[12-15,18]$. The primary aim of this systematic review was to summarise the current status and progress of MRI application in ILD. The most important articles published in the past two decades were reviewed to describe all possible applications of chest MRI in ILD. The review focuses on the potential role of MRI on ILD and emphasises the open questions that future research studies will have to address. Finally, we proposed an MRI protocol for ILD (M-ILD) to address these research questions.

\section{Methods}

A systematic literature search was conducted in collaboration with experienced medical librarians on the application of chest MRI in patients with ILD. We searched Embase, Medline, Web of Science, Scopus, Cochrane, Google scholar and PubMed databases for relevant publications. The last search was run on January 01, 2018. Key words included: chronic lung disease OR interstitial lung disease OR ILD OR UIP OR NSIP AND magnetic resonance imaging AND MRI. Titles and abstracts were screened by two independent reviewers (C. Romei and L. Turturici). We only included articles published in English that described original research and addressed chest MRI findings in patients with ILD. Reference lists of included articles were reviewed for additional references. 


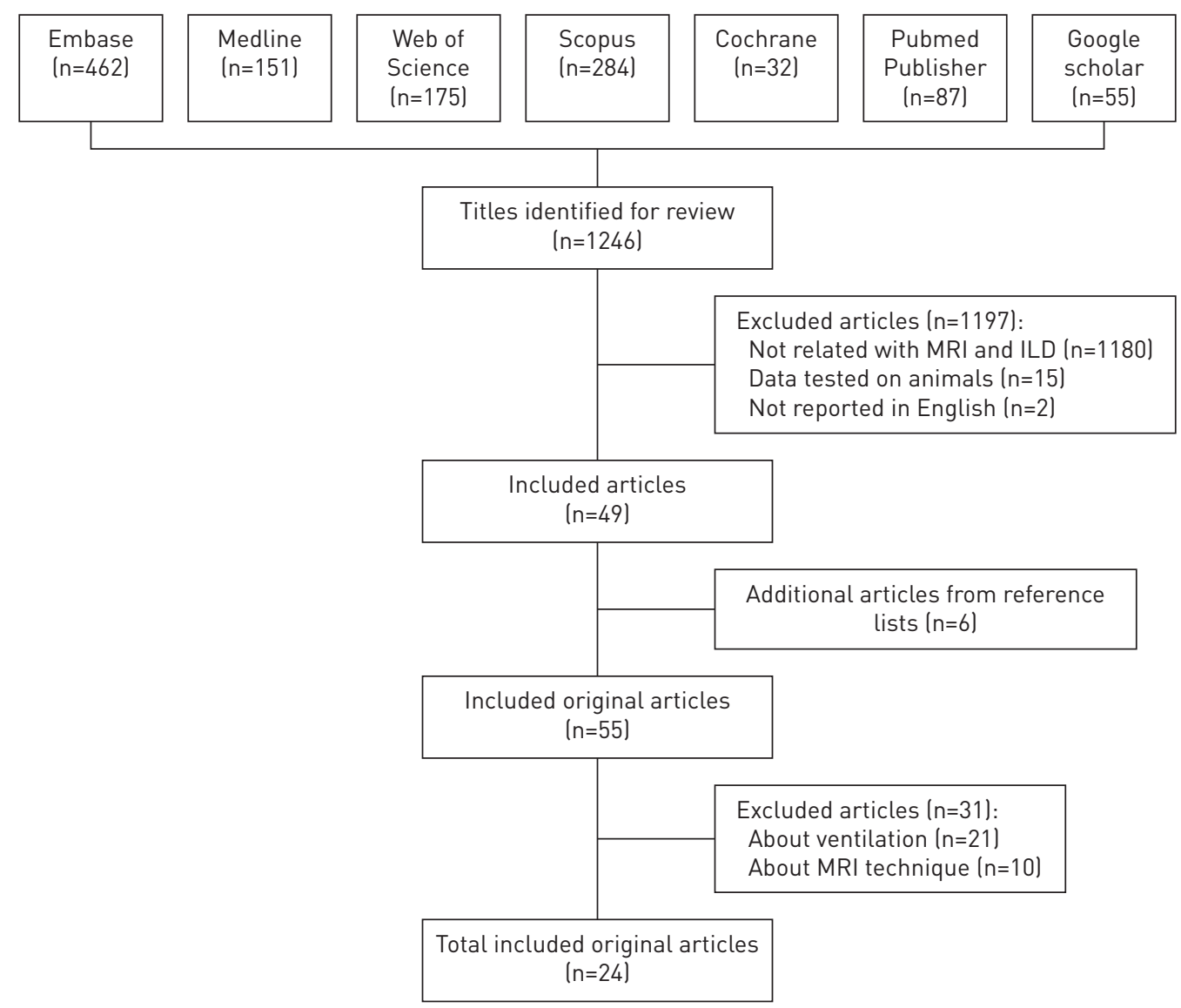

FIGURE 1 Flow chart of all the harvested papers. MRI: magnetic resonance imaging; ILD: interstitial lung disease.

\section{Results}

In total 1246 articles were retrieved. Figure 1 shows the flowchart for all harvested papers. Finally, 49 articles met the inclusion criteria and six additional articles were retrieved from their reference lists. We excluded 31 technical articles regarding specific chest MRI techniques. In total, we included 24 original articles (supplementary table 1). Year of publication varied from 1996 to 2017. Articles described several ILD patterns and groups of diseases using different diagnostic criteria for ILD. The results of these studies are grouped by MRI technique and presented according to their clinical availability, from those applicable in daily practice to the most advanced techniques performed in a research setting.

\section{Morphological imaging}

Thin-slices MDCT remains the gold standard to detect structural ILD abnormalities, such as ground-glass opacity, reticulation, honeycombing and traction bronchiectasis [9]. Thanks to the development of new MRI techniques, sequences have become available that can be useful for evaluating ILD abnormalities (figure 2) [18-20]. Several studies have compared sensitivity and specificity of thin-slices MDCT to MRI to assess ILD abnormalities [21]. The first sequence evaluated for morphological ILD imaging was a single-shot fast spin echo (Half-Fourier Single-shot Turbo Spin-Echo (HASTE); Siemens, Erlangen, Germany) T2-weighted acquisition. HASTE images provide high-signal intensity in water-rich tissues; therefore, lung abnormalities appear bright and surrounded by air-filled parenchyma with low-signal intensity. HATABU et al. [22] showed that breath-hold HASTE sequences were of diagnostic quality in 20 patients with several lung diseases, including interstitial abnormalities such as ground-glass opacity and bronchiectasis. Puderbach et al. [23] also used HASTE before and after contrast agent administration in patients with cystic fibrosis and demonstrated a moderate agreement with thin-slices MDCT. The concordance of chest MRI related to thin-slices MDCT to define the severity of bronchiectasis, peribronchial wall thickening, bullae and emphysema were $57 \%, 73 \%, 77 \%$ and $80 \%$, respectively [23]. Heкimoglu et al. [24] tested HASTE in patients with progressive massive fibrosis and found a significant agreement between MRI and thin-slices MDCT findings with an intraclass correlation coefficient (ICC) of 0.774 (single measures ICC). However, HASTE imaging did not show a high diagnostic quality because of 


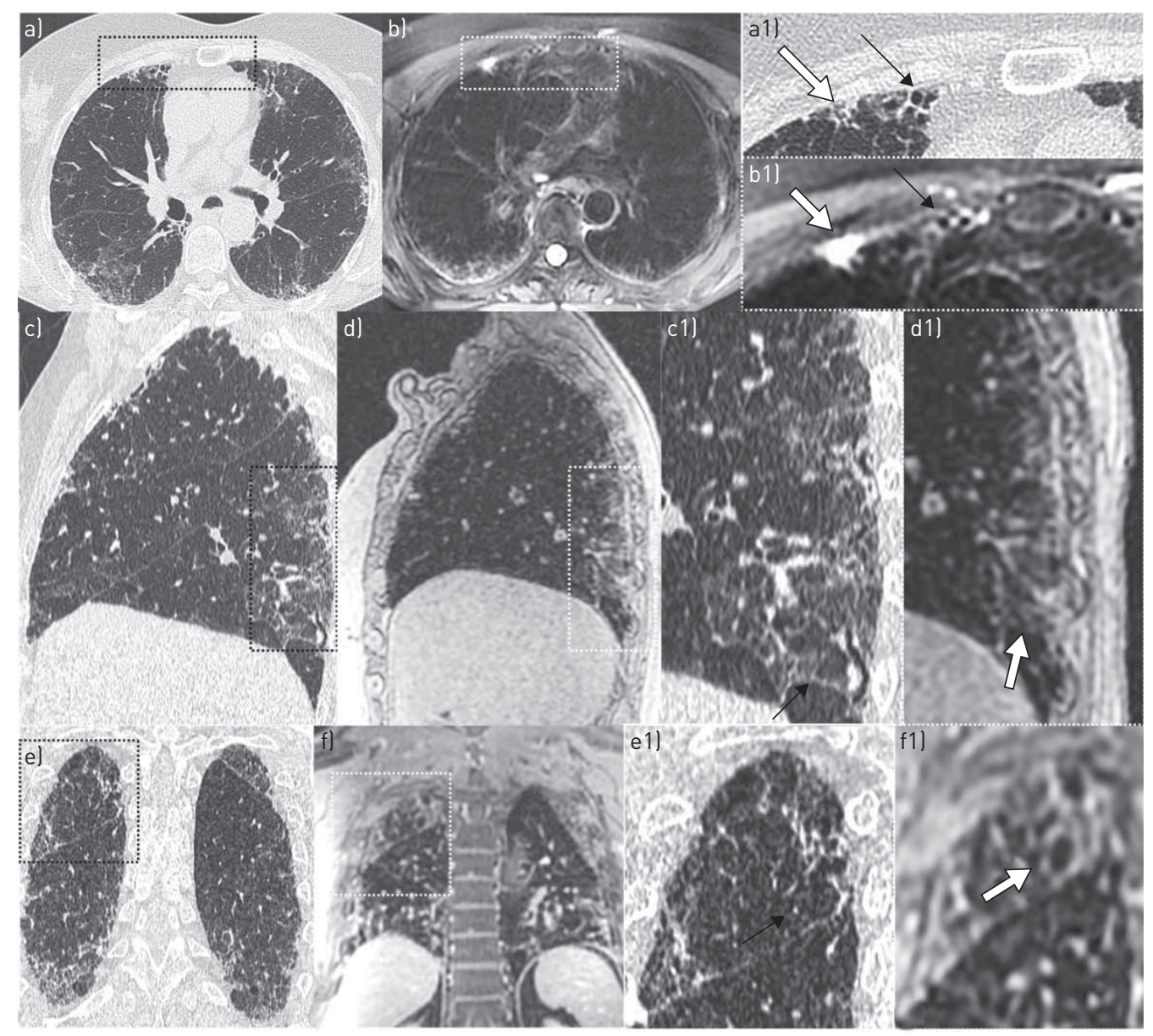

FIGURE 2 Comparison of radiological findings of interstitial lung disease with thin-slices multi-detector computed tomography (MDCT) and magnetic resonance imaging (MRI). Honeycombing at a) thin-slices MDCT and b) MRI two-dimensional axial PROPELLER T2-weighted sequence in a 78-year-old female with secondary usual interstitial pneumonia from collagen vascular disease. The magnified images of MDCT (a1) and MR (b1) show honeycombing (black arrow) and the area of ground-glass opacity (white arrow). The high signal of ground-glass opacity might be due to water content and therefore indicates active inflammation. Ground-glass opacity at c) thin-slices MDCT and d) MRI sagittal reformat three-dimensional SPGR proton density-weighted sequence pre-contrast in a 62-year-old male with idiopathic pulmonary fibrosis. On the magnified images of MDCT (c1) and MRI (d1), the ground-glass opacity is harder to identify on MRI (white arrow) than on MDCT (black arrow). Reticulation at e) thin-slices MDCT and f) MRI coronal reformat three-dimensional SPGR after T1-weighted contrast-enhanced administration (at $20 \mathrm{~min}$ ) in a 78-year-old female with secondary usual interstitial pneumonia from collagen vascular disease. In the magnified images of MDCT (e1) and MRI (f1) the different appearance of reticulation on MRI (white arrow) and MDCT (black arrow) is probably due to both a different breathing condition (MDCT inspiration versus MRI expiration) and slice thickness (MDCT $1 \mathrm{~mm}$ versus MRI $3 \mathrm{~mm}$ ).

respiratory and cardiac motion artefacts [24]. PInAL-Fernandez et al. [25] evaluated the utility of HASTE to assess the extent of ILD involvement in patients with systemic sclerosis. HASTE showed a good diagnostic performance to detect ILD with an area under the curve of 96\% (95\% CI 89-100\%). This study demonstrated that HASTE MRI could detect patients with $>0.5 \%$ ILD extent with a sensitivity of $93 \%$ and specificity of $100 \%$. Furthermore, MRI showed the same diagnostic performance $(p=0.03)$ of thin-slices MDCT to detect ILD in systemic sclerosis patients [25]. RAJARAM et al. [26] retrospectively analysed chest MRI of 224 patients, acquired using a two-dimensional balanced steady-state free-precession (bSSFP) sequence. bSSFP sequence provides a mixed weighting T2/T1 with fast acquisition that freezes respiratory and cardiac motion allowing acquisitions of the entire thorax in a single breath-hold [16]. Compared to thin-slices MDCT, the sensitivity and specificity of MRI in the identification of pulmonary fibrosis were 89\% (95\% CI 77-96\%) and 91\% (95\% CI 76-98\%). However, MRI only revealed $75 \%$ of ground-glass opacity, $67 \%$ of traction bronchiectasis and $45 \%$ of cystic reticular changes detected with thin-slices MDCT. MRI was even less effective in depicting emphysema (16\%) and minor fibrosis (67\%), although the diagnostic accuracy of MRI tended to increase in more advanced ILD stages. This study showed the 
utility of bSSFP in the evaluation of the severity of pulmonary fibrosis in an unselected group of patients. Although bSSFP and HASTE sequences were not directly compared, the authors underlined the advantages of bSSFP over HASTE due to a shorter echo and acquisition times, lower sensitivity to motion artefacts and mixed T2/T1 contrast weighting [23].

Other gradient echo-based sequences, such as the spoiled gradient echo sequences (SPGR; GE Healthcare, Milwaukee, WI, USA) and Volume Interpolated Breath-hold Examination (VIBE; Siemens), are usually performed as non-contrast acquisitions with a proton density weighting to enhance SNR. They are particularly suited to assess bulky consolidations and large areas of fibrosis in inspiration and to detect trapped air in expiration [16]. However, in the reviewed literature, we did not find any applications of this technique for ILD, but only in other small airways diseases [17]. T1-weighted gradient-echo imaging is usually combined with contrast administration to enhance parenchymal visualisation. In the reviewed literature, post-contrast T1-weighted imaging has been mostly used to assess pattern of enhancement of fibrotic tissue, therefore we will discuss it along with the post-contrast imaging techniques in a later section.

Currently, the most promising MRI sequences to evaluate lung abnormalities using MRI are the ultra-short echo-time sequences [27]. Ultra-short echo-time limits signal decay thanks to echo-time in the range of microseconds instead of milliseconds providing high SNR and high-resolution images. Image quality of ultra-short echo-time has been described to be almost comparable to thin-slices MDCT [28]. In a study of ОнNо et al. [12] ultra-short echo-time was compared to thin-slices MDCT to assess ILD findings in 19 patients. To diagnose ILD, ultra-short echo-time showed an overall sensitivity, specificity and accuracy of $100.0 \%, 97.0 \%$ and $97.6 \%$, respectively. However, to evaluate each abnormality individually, such as reticulation, bronchiectasis, ground-glass opacity and honeycombing, thin-slices MDCT showed higher sensitivity, specificity and accuracy than ultra-short echo-time. For instance, to define reticulation, thin-slices MDCT has a sensitivity, specificity and accuracy of $87.2 \%, 96.4 \%$ and $95.6 \%$, respectively, compared to ultra-short echo time that was $71.4 \%, 94.4 \%$ and $92.8 \%$. A current limitation of ultra-short echo-time is the data acquisition in free-breathing end-respiratory conditions, which can determine underestimation of the severity of airways disease in ILD patients. With thin-slices MDCT, ILD is routinely assessed with end-inspiratory breath-hold scans in supine and prone positions, for a better definition of reticulation and honeycombing [29]. These different breathing conditions could have contributed to the different performance of ultra-short echo-time compared to thin-slices MDCT in the study of OHNo et al. [12]. To date, ultra-short echo-time has only been tested in research settings, therefore larger validation studies with multicentre ILD cohorts are needed to confirm these promising results [12].

\section{Functional imaging}

Although thin-slices MDCT remains superior to MRI to assess structural changes in ILD, several functional MRI techniques might play a complementary role in ILD. Ventilation techniques without and with gaseous contrasts could provide important information regarding ventilation mismatch in ILD patients [30]. Active lung inflammatory tissue could be assessed with T2-weighted sequences or using diffusion weighted imaging (DWI) [31]. Perfusion studies with non-contrast and contrast-enhanced MRI techniques could be used to quantify the amount of lung fibrosis [13]. Eventually, dynamic MRI could have a role in quantifying diaphragm mechanics and progressive lung fibrosis of ILD patients [16].

These techniques will be briefly described below, starting with those clinically available to the most experimental techniques. When available, results of studies using the described MRI technique will be presented.

\section{Ventilation}

For direct lung ventilation imaging with magnetic resonance, several techniques are available. They either rely on oxygen enhancement, hyperpolarised noble gases or Fourier decomposition [32-34]. The oxygen-enhanced MRI can be applied to assess regional ventilation, alveolar-capillary gas transfer of molecular oxygen, oxygen uptake per respiratory cycle and airflow limitation $[35,36]$. This method uses the paramagnetic effect of pure oxygen to shorten T1 relaxation times, leading to a signal enhancement [37]. Several studies have tested oxygen-enhanced MRI in patients with ILD. Molinari et al. [32] demonstrated a statistically significant correlation between oxygen-enhanced MRI and PFTs in patients with UIP $(n=1)$, NSIP $(n=8)$ and sarcoidosis $(n=1)$. STADLER et al. [38] showed a shortening of the T1 relaxation time in patients with lung fibrosis, suggesting that $\mathrm{T} 1$ measurements could be used to monitor disease progression and quantify the amount of lung fibrosis. Furthermore, STADLER et al. [39, 40], using T1 maps, investigated how the breathing status influenced T1 values of the lung parenchyma and demonstrated a statistically significant difference of inspiratory $\mathrm{T} 1$ values in comparison to expiratory ones both in healthy individuals [39] and patients with emphysema and fibrosis [40]. Moreover, both 
pathological groups showed lower average T1 values in inspiration and expiration compared to healthy individuals while the average expiratory $\mathrm{T} 1$ was significantly higher in the fibrosis group than the emphysema group [40].

OHNo et al. [41] found that oxygen-enhanced MRI was comparable to thin-slices MDCT to determine pulmonary functional loss and disease severity in ILD patients with connective tissue disease. Oxygen-enhanced MRI is a cheap and safe technique, but so far it has been performed in small groups of patients and in research settings, because, it remains quite challenging with long scan acquisitions and poor SNR [42]. Hyperpolarised gas-MRI, using helium $\left({ }^{3} \mathrm{He}\right)$ or xenon $\left({ }^{129} \mathrm{Xe}\right)$ as gaseous contrasts provides quantitative regional information on pulmonary ventilation and lung microstructure changes [43]. Currently, hyperpolarised gas-MRI has not been used in ILD. Hyperpolarised gas-MRI provides high-image quality and high SNR $[42,44]$. Despite its established role for imaging lung function, the complicated process of hyperpolarisation of the noble gases, the high costs, in particular for ${ }^{3} \mathrm{He}$, and the need of dedicated hardware has, so far, precluded the translation of this technology to the clinic [42]. Hopefully in the future, hyperpolarised xenon-MRI will be made available as a diagnostic and therapeutic monitoring tool for lung-related diseases, including ILD. To date, no studies have been conducted in ILD with fluorinated gas MRI, a new technique that provides high-quality ventilation imaging similar in quality to those from hyperpolarised gas-MRI.

\section{Contrast-enhanced techniques}

Contrast-enhanced thin-slices MDCT is not routinely used in ILD, because iodine contrast does not increase the sensitivity or specificity to detect and characterise ILD abnormalities. However, contrast determines a significant increase of radiation exposure in patients [45].

Contrast-enhanced magnetic resonance perfusion, thanks to its ability to characterise different tissues, could represent a potential alternative in clinical practice. MRI for myocardial fibrosis detection, using specific late contrast-enhanced sequences, is a well-validated technique routinely used in cardiac imaging. As matter of fact, gadolinium-based contrast agent (GBCA) washes out from normal tissue while it remains in fibrotic tissue. This characteristic was used by LAVelle et al. [46] to detect and characterise pulmonary fibrosis. In this study, a specific double inversion-recovery gradient echo-pulse sequence was used to null the pulmonary arterial blood signal and depict pulmonary fibrosis with late-enhanced MRI. LAVELLE et al. [46] demonstrated that the extent of pulmonary fibrosis on late-enhanced MRI correlated significantly with thin-slices MDCT $(\mathrm{r}=0.78, \mathrm{p}<0.001)$. However, the percentage of reticulation or honeycombing showed no significant correlations between the two modalities $(\mathrm{p}=0.34$ and $\mathrm{p}=0.23$, respectively). MirSADRAEE et al. [47] evaluated T1 signal characteristics in the fibrotic lung parenchyma pre- and post-contrast injection at 10 and $20 \mathrm{~min}$. At $10 \mathrm{~min}$ after contrast injection, normal lung of healthy volunteers had a significantly longer T1 than both fibrotic and morphologically normal lung in patients with ILD. T1 of fibrotic lung tissue continued to decrease until $20 \mathrm{~min}$ after contrast injection, whereas morphologically normal lung T1 did not significantly change after $10 \mathrm{~min}$. These results showed a different contrast enhancement between healthy lung tissue and morphologically normal lung in ILD patients (figure 3). Therefore, GBCA could be used to identify early fibrotic changes (not yet detectable by thin-slices MDCT) in morphologically normal lung parenchyma.

KING et al. [48] evaluated the effects of a tailored window setting for lung MRI and gadopentetate dimeglumine (Magnevist; Bayer, Berlin, Germany) on the visibility of pulmonary abnormalities occurring in UIP. In this study T1-weighted MRIs were obtained before and after contrast administration and visualised with conventional window setting and with "MRI lung" window setting chosen to increase the visibility of lung parenchyma, similar to a lung window setting on thin-slices MDCT. The use of GBCA significantly improved the detection of honeycombing (sensitivity $59 \%$ and specificity $89 \%$ ), but it did not influence the detection of ground-glass opacity. The use of a MRI lung window setting improved the detection of ground-glass opacity both in the contrast-enhanced and unenhanced scans. Moreover, MRI lung window setting improved the detection of honeycombing in the contrast-enhanced scans.

In the study of HeкimoĞLu et al. [24], two fast imaging sequences (HASTE and VIBE) were tested with and without GBCA for evaluating progressive massive fibrosis. Interestingly, post-contrast VIBE imaging showed the best agreement (ICC 0.999, single measures) with thin-slices MDCT, indicating a better detection of ILD features after GBCA administration. To date, perfusion imaging with dynamic contrast enhancement technique has been tested in the aforementioned studies and in studies to assess disease activity, which will be discussed in the following sections. 

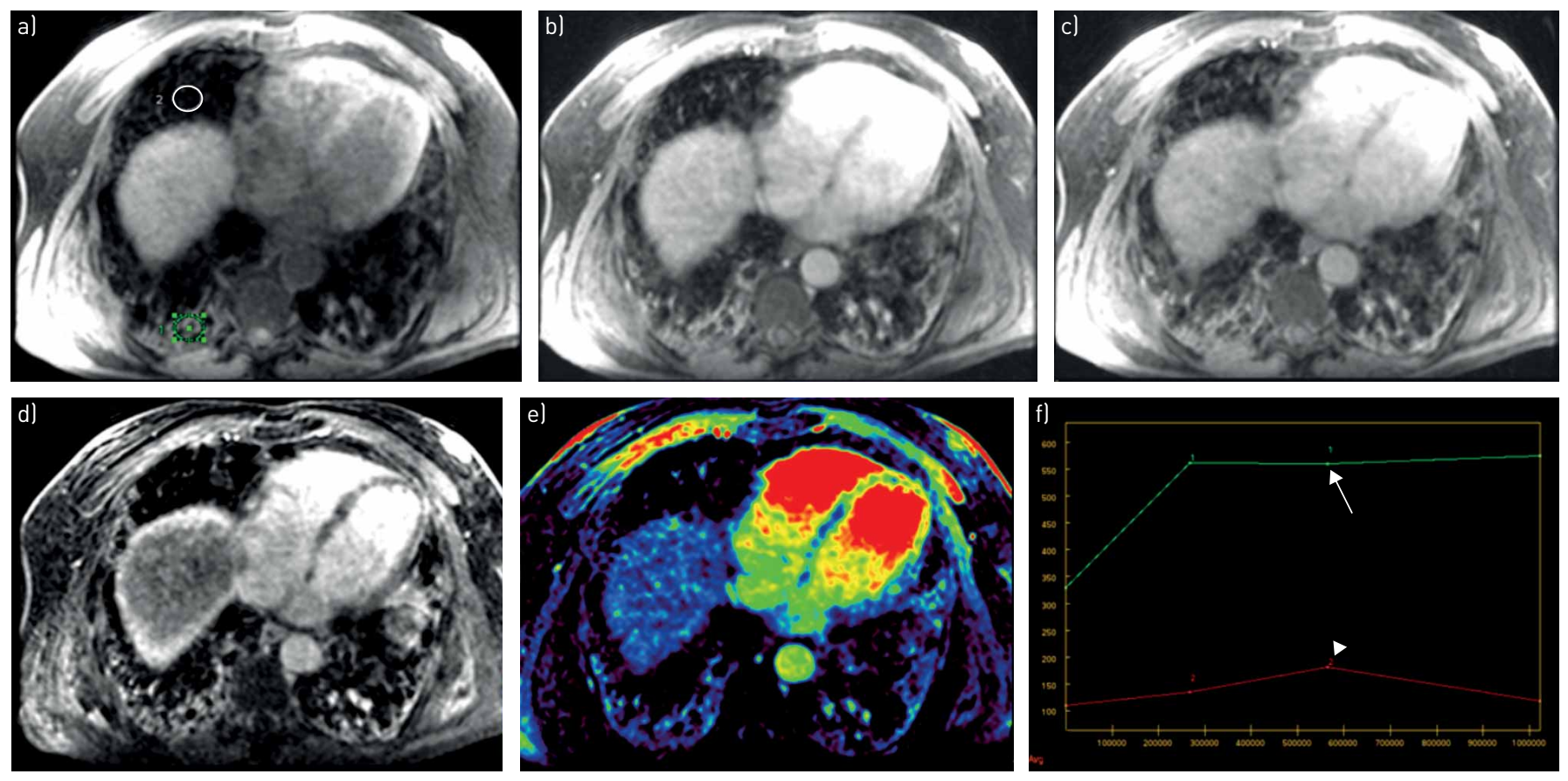

FIGURE 3 T1-contrast enhancement signal characteristics in the fibrotic and normal lung parenchyma pre- and post-contrast injection at $\sim 5,10$ and $20 \mathrm{~min}$ in a 73-year-old female with fibrotic nonspecific interstitial pneumonia. a) Chest magnetic resonance imaging axial reformat three-dimensional SPGR proton density-weighted pre-contrast. Axial reformats three-dimensional SPGR T1-weighted post-contrast at b) 5 min and c) $10 \mathrm{~min}$. d) Contrast enhancement subtraction image (10 min image subtracted to pre-contrast). e) Axial maximum slope of increase of contrast enhancement. f) Contrast enhancement curves for fibrotic and normal lung at different time-points, Green region of interest in (a) and arrow in ( $\mathrm{f}$ ) indicate fibrotic lung tissue, while white region of interest in (a) and arrowhead in ( $\mathrm{f}$ ) indicate normal lung tissue. Note progressive contrast enhancement of fibrotic lung with peak at 10 min (arrow), while normal lung has a lower contrast enhancement with signal decay after the first 5 min (arrowhead).

disease progression [49]. Other progressive forms of pulmonary fibrosis, such as collagen vascular disease-related ILD, are treated with anti-inflammatory drugs. Several on-going trials are currently recruiting patients with (progressive) pulmonary fibrosis other than IPF including collagen vascular disease (www.clinicaltrials.gov NCT02999178 and NCT02597933; and DRKS00009822). In this clinical setting, the ability to assess the disease activity and, in particular, to detect lung areas with active inflammation and early fibrosis might be a crucial parameter to determine the type of treatment (e.g. antifibrotic versus anti-inflammatory) and to monitor its efficacy. This ability is lacking with thin-slices MDCT, which is extremely limited to distinguish active inflammation from fibrosis. In fact, fibrotic lung tissue with and without active lung inflammation could have similar appearance with ground-glass opacity and reticulation [50]. Conversely the higher tissue characterisation of MRI could represent an innovative and noninvasive method to assess disease activity of ILD.

\section{Conventional sequences: single shot, fast spin echo}

LUTTERBEy et al. [51] evaluated the feasibility of lung MRI at high magnetic field strengths (3.0T) using a fat suppressed (Fat-Sat) T2 Fast Spin Echo sequence to assess active versus non-active lung disease in ILD patients. This sequence is extremely sensitive to fluids. Since inflammation is accompanied by higher water content in the inflammatory tissue, they hypothesised a higher T2-weighted signal in active inflammatory lung lesions compared to chronic and fibrotic lung tissue. The results of their study showed that normal lung tissue appeared long T2 component signal free, that of fibrotic tissue was comparable to the muscle signal (intermediate signal), while inflammatory tissue showed high T2 (water-like) signal. Signal intensity was calculated by placing the region-of-interest in multiple lung areas with abnormalities and with different T2 signal intensity. Thin-slices MDCT was used as a reference standard to correlate morphological changes. Those lung areas with signal intensity twice higher that of the intermediate-signal areas were the lung area having the typical features of active ILD disease, such as ground-glass opacity. These findings were also confirmed by pathological evaluation obtained on lung biopsy specimen.

\section{T2 mapping}

BUZAN et al. [52] compared the T2 relaxation time of normal and impaired lung tissue. By using T2 quantitative mapping, they characterised and differentiated ground-glass opacity, reticulation and 
honeycombing in UIP and NSIP [52]. T2 sagittal maps were generated using a multi-echo single-shot, turbo spin echo sequence. They found that the median T2 relaxation time of normal lung was $41 \mathrm{~ms}$ and was significantly shorter than in pathological areas $(\mathrm{p}<0.001)$. Moreover, T2 relaxation time was significantly different $(\mathrm{p}<0.05)$ between ground-glass opacity, reticulation and honeycombing with a median value of $66.5 \mathrm{~ms}, 74.3 \mathrm{~ms}$ and $79.5 \mathrm{~ms}$, respectively. This study demonstrated that T2 relaxation time tended to increase significantly with progression of fibrotic changes in ILD, the authors explained that the increase of water-rich tissues and macromolecule fraction in fibrosis leads to increased T2 relaxation time, which depends on water content and its interaction with macromolecules.

\section{Diffusion weighted imaging}

DWI is a noninvasive technique for measuring the water diffusion rate within tissue. Water diffusion is frequently altered in various disease processes and may reflect physiological and morphological characteristics, such as cell density and tissue viability. DWI has been used for lung cancer imaging [53] and more recently to detect and quantify inflammation in cystic fibrosis patient with a pulmonary exacerbation [54]. The latter application is of particular interest for ILD patients because it could be used as a potential marker of active disease and to monitor treatment response (figure 4) [31]. To date, DWI has not been tested on ILD patients. Future studies should test feasibility of DWI on ILD patients.

\section{Contrast-enhanced techniques}

YI et al. [13] studied the utility of 3.0T contrast-enhanced MRI for differentiating inflammation and fibrosis predominant lesions in the UIP and NSIP. Contrast-enhanced MRI consisted of T1-weighted three-dimensional turbo field-echo sequence using an intravenous bolus injection of gadopentetate dimeglumine $\left(0.2 \mathrm{~mL} \cdot \mathrm{kg}^{-1}\right.$ of Magnevist). Enhancement pattern was visually assessed prospectively and classified into three categories using the dynamic contrast images: pattern 1: early enhancement and washout with discernible enhancement of peak enhancement at 1 or $3 \mathrm{~min}$; pattern 2: slight enhancement with no discernible enhancement at a specific time-point throughout dynamic phases; and pattern 3: delayed persistent enhancement with discernible peak enhancement at 5 or $10 \mathrm{~min}$. Qualitatively analysis showed that $82 \%$ of inflammation-predominant lesions exhibited early enhancement (pattern 1) and that 94\% of fibrosis-predominant lesions exhibited slight enhancement (pattern 2) or delayed persistent enhancement (pattern 3). The frequency of pattern 1 was significantly higher in the inflammation-predominant lung lesions (82\% confidence interval). Quantitative assessment of dynamic enhanced MRIs were obtained by measuring the mean signal intensity of the lung lesions with regions-of-interest positioned by a radiologist in correlation to the thin-slices MDCT findings. The following parameters were evaluated: maximum peak enhancement; time to peak; slope of enhancement; and extent of washout. Inflammation-predominant lesions sites had higher percentage signal intensity at $1 \mathrm{~min}$, shorter time to peak and faster slope of enhancement than fibrosis-predominant sites. In summary, qualitative analysis of dynamic T1-weighted three-dimensional turbo field-echo MRIs obtained at 3.0T proved helpful for differentiating inflammation- and fibrosis-predominant lesions.

GAETA et al. [55] evaluated the gadolinium-enhanced MRI in the assessment of disease activity in 25 consecutive patients with chronic infiltrative lung diseases. They assumed that gadolinium enhancement might correlate with disease activity, because pulmonary insults inducing lung fibrosis disrupt capillary endothelium and permit the extravasation of contrast into the interstitial and alveolar spaces. Two radiologists retrospectively evaluated MRI and analysed the studies for the presence (group 1) or absence (group 2) of pulmonary lesion enhancement. The presence of enhancement was considered predictive of active inflammation; the absence of enhancement was considered predictive of inactivity. The presence of enhanced pulmonary lesions was seen in 14 out of 17 patients with active disease. Negative enhancement was seen in all eight (100\%) patients with inactive disease, and in three (18\%) out of 17 patients with active disease. In both groups the difference was statistically significant (Fisher exact test, $\mathrm{p}<0.05$ ). These data showed that the presence of enhancing lesions on gadolinium-enhanced T1-weighted MRI studies may be a reliable indicator of inflammation and, consequently, potentially influence treatment choice and follow-up.

\section{Phase-contrast MRI}

ILD may be complicated by pulmonary hypertension from pulmonary vasospasm due to: hypoxaemia, pulmonary vasoconstriction and vascular remodelling, vascular destruction associated with progressive parenchymal fibrosis, vascular inflammation, perivascular fibrosis and thrombotic angiopathy. Although echocardiography is the mainstay for imaging of the right heart in clinical practice, advances in velocity-encoded MRI as well as other cardiac magnetic resonance techniques have provided an opportunity for quantitative and qualitative assessment of haemodynamics in the pulmonary circulation and identification of right ventricular morphological changes. Phase-contrast MRI combined with cardiac MRI is now regarded as the reference standard for the assessment of right ventricle structure and function [31]. 


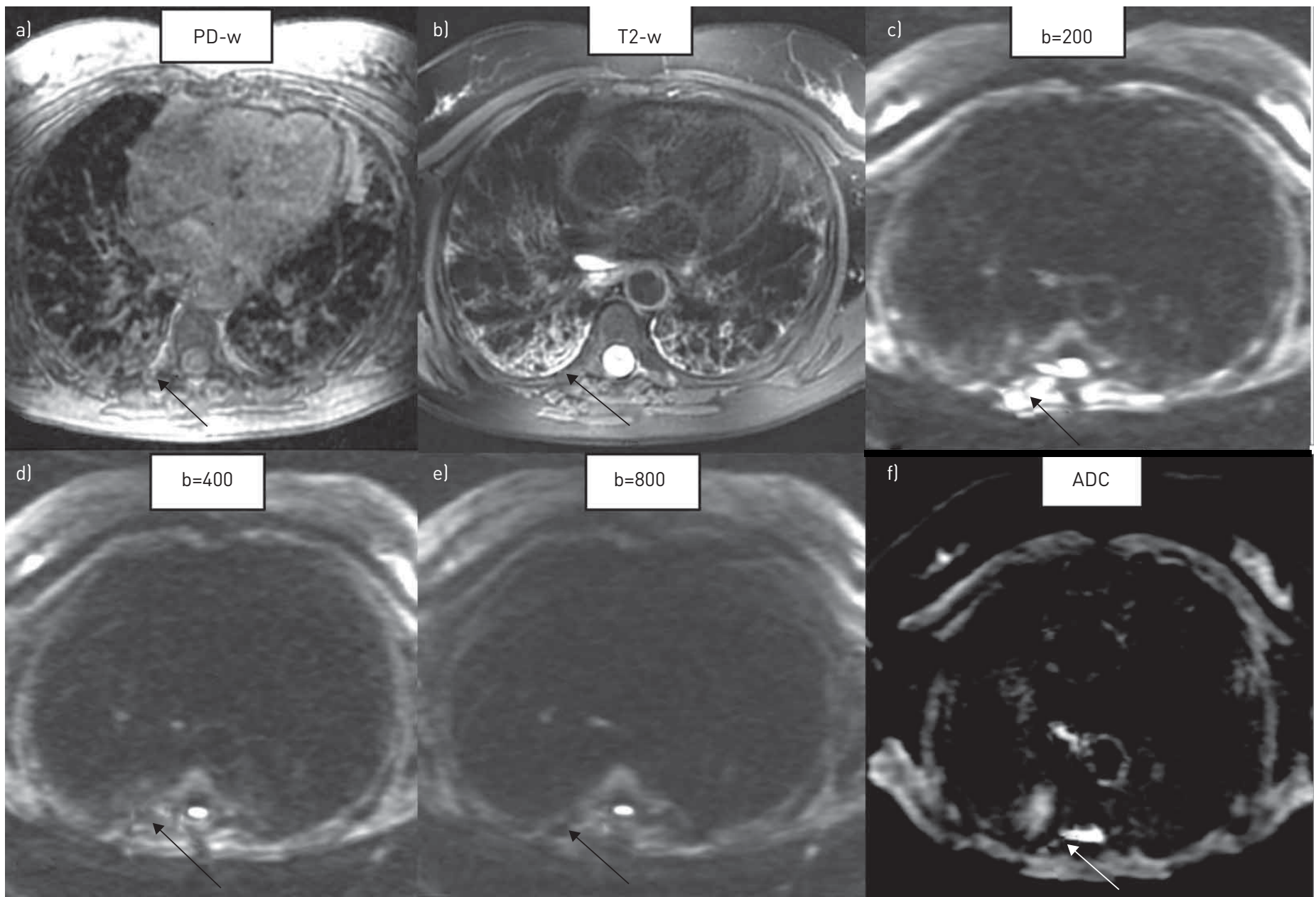

FIGURE 4 Diffusion weighted imaging (DWI) at multiple b-values in a 72-year-old female with nonspecific interstitial pneumonia. a) Three-dimensional SPGR proton density-weighted (PD-w) image, b) two-dimensional fat suppressed PROPELLER T2-weighted image, c-el DWl at b-values c) 200 , d) 400 and el $800 \mathrm{~s} \cdot \mathrm{mm}^{-2}$, and f) apparent diffusion coefficient (ADCl map. Note area of inflammation in proton density-weighted image (arrow in al, which has a high T2-weighted signal (arrow in b) and shows signal decay by increasing b-values in the DWI images larrows in c-e). In the apparent diffusion coefficient maps, the same area in the right lower lobe is hyperintense possibly indicating either high water content or increased perfusion. 
Nowadays, phase-contrast MRI is a standard subset of cardiac MRI protocols. Phase-contrast MRI was tested on ILD patients to correlate the decrease of pulmonary blood flow detected by phase-contrast MRI (measured in the main, right and left pulmonary arteries) with the decrease in lung volume and increase of pulmonary fibrosis calculated by thin-slices MDCT. The study showed that progression of lung fibrosis is associated with pulmonary blood flow reduction in ILD with or without pulmonary hypertension. It was speculated that this finding was due to increased resistance in the pulmonary arterioles $[56,57]$.

\section{Cine MR}

Chest mechanics using MRI has been investigated in several chronic obstructive lung diseases [58, 59]. It has been shown that diaphragmatic function correlates well with lung function in asthma and chronic obstructive pulmonary disease patients. Chest mechanics and diaphragm function is also particularly important in neuromuscular diseases, where diaphragmatic function is monitored to assess treatment efficacy [60]. To date, however, chest mechanics has not been investigated in ILD patients. Lung fibrosis is associated with a reduction of the elastic recoil and consequently higher workload for the respiratory muscles to expand the chest cage, which eventually results in lower lung volumes [2]. Theoretically, disease progression in fibrotic ILD patients should demonstrate a reduction of diaphragm function. This information could be used to monitor worsening of lung function in ILD patients and possibly used as a new outcome measure for prognosis and indication to lung transplantation.

The development of progressive parenchymal fibrosis in ILD patients causes lung restriction as a result of increased lung stiffness and reduced compliance. Recently, MARINelLi et al. [61] investigated the use of magnetic resonance elastography in the quantitative assessment of pulmonary fibrosis by comparing quantitative shear stiffness measurements of lung parenchyma in patients with a diagnosis of ILD and normal controls. A $1.5 \mathrm{~T}$ two-dimensional spin-echo, echo planar imaging magnetic resonance elastography pulse sequence was utilised to assess absolute lung shear stiffness in 15 patients diagnosed with ILD and in 11 healthy controls. Lung shear stiffness was evaluated at residual volume and total lung capacity (TLC). Prior to scanning all patients underwent spirometry. Patients with ILD exhibited an average shear stiffness of $2.74 \mathrm{kPa}$ at TLC and $1.32 \mathrm{kPa}$ at residual volume. The corresponding values for healthy individuals were $1.33 \mathrm{kPa}$ and $0.849 \mathrm{kPa}$, respectively. The difference in shear stiffness between residual volume and TLC was statistically significant $(\mathrm{p}<0.001)$. This study demonstrated that the shear stiffness in patients with ILD, measured by magnetic resonance elastography, is increased when compared to healthy individuals at both residual volume and TLC. Because in this study the lung stiffness increases in ILDs with increasing transpulmonary pressure (i.e. from residual volume to TLC), the most significant difference in shear stiffness was demonstrated at TLC.

\section{Proposed MRI protocol}

Based on the findings in the literature, we have developed a patient-friendly MRI ILD protocol (M-ILD). Our M-ILD protocol was developed using a 3.0T whole body MRI system (Discovery MR750 3.0T; GE Healthcare). We use an eight-element phased array cardiac coil with the patient in the supine position and breathing monitoring using a respiratory belt. ILD patients have problems of hyperpnoea and cannot lie down for a long time. Therefore, we elaborated a short MRI scan protocol with pre-contrast acquisitions of $\sim 20 \mathrm{~min}$, followed by a scan break and post-contrast acquisitions at 10 and $20 \mathrm{~min}$. To increase protocol feasibility, we used commercially available sequences, limiting the use of experimental MRI techniques that are only available in the research setting. The proposed M-ILD protocol is summarised in supplementary table 2 with optional sequences for a research setting.

For morphological analysis, we proposed a three-dimensional proton density-weighted (PD-w) sequence (3D SPGR), and a two- and three-dimensional T2-w acquisition (2D fat suppressed (FS) PROPELLER and 3D CUBE T2 FS).

For functional imaging we used a 3D SPGR T2* multi-echo sequence both in inspiration and expiration to generate $\mathrm{T}^{*}$ maps. $2^{*}$ maps could be used to assess lung fibrosis and ventilation (figure 5). We also added a 2D DWI multi b-value acquisition to assess lung inflammation. Finally, we added 3D SPGR FS sequences before and after injection of $0.2 \mathrm{mmol} \cdot \mathrm{kg}^{-1}$ of gadobenate dimeglumine (MultiHance; Bracco, Milan, Italy) at 10 and $20 \mathrm{~min}$ to evaluate late enhancement of fibrotic lung tissue. Post-processing analysis of the data was also performed with commercially available software on the AW Server 2.0 platform (GE Healthcare).

We aimed to test our M-ILD protocol in a large multicentre cohort study in patients with ILD. For a multicentre study, robust and feasible MRI techniques are needed. The M-ILD protocol has been developed with commercially available sequences, which can be implemented with all MRI brands. Image quality standardisation between centres can be achieved using a dedicated lung-MRI phantom [62]. However, some of the proposed sequences are still high demanding and complex techniques, such as 

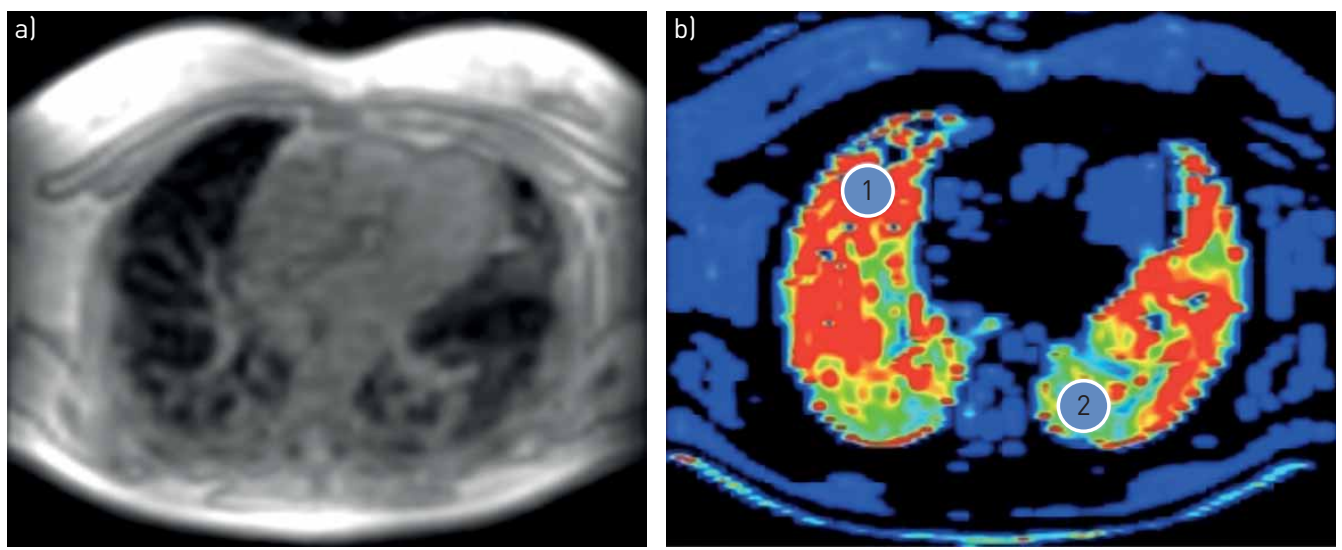

FIGURE 5 T2 mapping. a) Native axial multi-echo three-dimensional SPGR in a 72-year-old female with nonspecific interstitial pneumonia. b) Post-processed R2* map $\left(R 2^{*}=1 / T 2^{*}\right)$ axial with region of interest 1 in normal lung tissue $(2395 \mathrm{~Hz}=0.42 \mathrm{~ms})$ and region of interest 2 in fibrotic lung tissue $(887 \mathrm{~Hz}=1.13 \mathrm{~ms})$. Note how T2* is longer in fibrotic tissue, therefore R2 is smaller (low signal-to-noise ratio) compared to normal tissue. Post-processing performed with Advantage Window Server 2.0 (GE Healthcare, Milwaukee, WI, USA).

hyperpolarised gas-MRI, oxygen-enhanced MRI, T1/T2 mapping that could require further refinement before large scale application.

\section{Future directions}

The role of thin-slices MDCT to evaluate progression and treatment response of ILD is still debated, because of the poor reproducibility of visual score for several parenchymal abnormalities and lack of specific outcome measures to evaluate response to treatment.

Although image resolution of MRI does not yet match that of thin-slices MDCT, MRI could play an important role for functional imaging in patients with ILD. In particular, the ability of MRI to detect and quantify inflammatory changes using different tissue signal weighting could be used to monitor treatment efficacy of new ILD drugs. MRI could differentiate between active inflammatory and fibrotic changes thus guiding targeted therapy in patients with ILD. These hypotheses need to be proved in future studies in order to determine whether MRI could have a role in the management of patients with ILD. Functional MRI could fill the diagnostic gaps still present in thin-slices MDCT imaging of ILD patients.

Conflict of interest: C. Romei has nothing to disclose. L. Turturici has nothing to disclose. L. Tavanti has nothing to disclose. J. Miedema has nothing to disclose. S. Fiorini has nothing to disclose. M. Marletta has nothing to disclose. P. Wielopolski has nothing to disclose. H. Tiddens reports industry funding from Roche, lecture and advisory board fees from Novartis, and grants from CFF, Vertex, Gilead and Chiesi, outside the submitted work. In addition, H. Tiddens has a patent licensed with Vectura, and a patent PRAGMA-CF scoring system issued. F. Falaschi has nothing to disclose. P. Ciet reports personal fees from Vertex Pharmaceutical, outside the submitted work.

\section{References}

1 Travis WD, Costabel U, Hansell DM, et al. An official American Thoracic Society/European Respiratory Society statement: update of the international multidisciplinary classification of the idiopathic interstitial pneumonias. Am J Respir Crit Care Med 2013; 188: 733-748.

2 Raghu G, Collard HR, Egan JJ, et al. An official ATS/ERS/JRS/ALAT statement: idiopathic pulmonary fibrosis: evidence-based guidelines for diagnosis and management. Am J Respir Crit Care Med 2011; 183: 788-824.

3 Flaherty KR, King TE, Raghu G, et al. Idiopathic interstitial pneumonia: what is the effect of a multidisciplinary approach to diagnosis. Am J Respir Crit Care Med 2004; 170: 904-910.

4 Aziz ZA, Wells AU, Bateman ED, et al. Interstitial lung disease: effects of thin-section CT on clinical decision making. Radiology 2006; 238: 725-733.

5 Hansell DM, Bankier AA, MacMahon $\mathrm{H}$, et al. Fleischner Society: glossary of terms for thoracic imaging. Radiology 2008; 246: 697-722.

6 Hewitt MG, Miller WT, Reilly TJ, et al. The relative frequencies of causes of widespread ground-glass opacity: a retrospective cohort. Eur J Radiol 2014; 83: 1970-1976.

7 Oldham JM, Noth I, Martinez FJ. Pharmacogenetics and interstitial lung disease. Curr Opin Pulm Med 2016; 22: 456-465.

8 Flaherty KR, Kolb M, Vancheri C, et al. Stability or improvement in forced vital capacity with nintedanib in patients with idiopathic pulmonary fibrosis. Eur Respir J 2018; 52: 1702593.

9 Walsh SL, Calandriello L, Sverzellati N, et al. Interobserver agreement for the ATS/ERS/JRS/ALAT criteria for a UIP pattern on CT. Thorax 2016; 71: 45-51. 
10 Jacob J, Bartholmai BJ, Rajagopalan S, et al. Serial automated quantitative CT analysis in idiopathic pulmonary fibrosis: functional correlations and comparison with changes in visual CT scores. Eur Radiol 2018; 28: 1318-1327.

11 Hansell DM, Goldin JG, King TE, et al. CT staging and monitoring of fibrotic interstitial lung diseases in clinical practice and treatment trials: a position paper from the Fleischner Society. Lancet Respir Med 2015; 3: 483-496.

12 Ohno Y, Koyama H, Yoshikawa T, et al. Pulmonary high-resolution ultrashort TE MR imaging: comparison with thin-section standard- and low-dose computed tomography for the assessment of pulmonary parenchyma diseases. J Magn Reson Imaging 2016; 43: 512-532.

13 Yi CA, Lee KS, Han J, et al. 3-T MRI for differentiating inflammation- and fibrosis-predominant lesions of usual and nonspecific interstitial pneumonia: comparison study with pathologic correlation. AJR Am J Roentgenol 2008; 190: 878-885.

14 Barreto MM, Rafful PP, Rodrigues RS, et al. Correlation between computed tomographic and magnetic resonance imaging findings of parenchymal lung diseases. Eur J Radiol 2013; 82: e492-e501.

15 Tiddens HA, Stick SM, Wild JM, et al. Respiratory tract exacerbations revisited: ventilation, inflammation, perfusion, and structure (VIPS) monitoring to redefine treatment. Pediatr Pulmonol 2015; 50: Suppl. 40, S57-S65.

16 Ciet P, Tiddens HA, Wielopolski PA, et al. Magnetic resonance imaging in children: common problems and possible solutions for lung and airways imaging. Pediatr Radiol 2015; 45: 1901-1915.

17 Wild JM, Marshall H, Bock M, et al. MRI of the lung (1/3): methods. Insights Imaging 2012; 3: 345-353.

18 Biederer J, Mirsadraee S, Beer M, et al. MRI of the lung (3/3): current applications and future perspectives. Insights Imaging 2012; 3: 373-386.

19 Biederer J, Beer M, Hirsch W, et al. MRI of the lung (2/3): Why...when...how? Insights Imaging 2012; 3 355-371.

20 Hochhegger B, Marchiori E, Irion K, et al. Magnetic resonance of the lung: a step forward in the study of lung disease. J Bras Pneumol 2012; 38: 105-115.

21 Biederer J, Both M, Graessner J, et al. Lung morphology: fast MR imaging assessment with a volumetric interpolated breath-hold technique: initial experience with patients. Radiology 2003; 226: 242-249.

22 Hatabu H, Gaa J, Tadamura E, et al. MR imaging of pulmonary parenchyma with a half-Fourier single-shot turbo spin-echo (HASTE) sequence. Eur J Radiol 1999; 29: 152-159.

23 Puderbach M, Eichinger M, Gahr J, et al. Proton MRI appearance of cystic fibrosis: comparison to CT. Eur Radiol 2007; 17: 716-724

24 Hekimoğlu K, Sancak T, Tor M, et al. Fast MRI evaluation of pulmonary progressive massive fibrosis with VIBE and HASTE sequences: comparison with CT. Diagn Interv Radiol 2010; 16: 30-37.

25 Pinal-Fernandez I, Pineda-Sanchez V, Pallisa-Nuñez E, et al. Fast 1.5 T chest MRI for the assessment of interstitial lung disease extent secondary to systemic sclerosis. Clin Rheumatol 2016; 35: 2339-2345.

26 Rajaram S, Swift AJ, Capener D, et al. Lung morphology assessment with balanced steady-state free precession MR imaging compared with CT. Radiology 2012; 263: 569-577.

27 Ohno Y, Nishio M, Koyama H, et al. Pulmonary MR imaging with ultra-short TEs: utility for disease severity assessment of connective tissue disease patients. Eur J Radiol 2013; 82: 1359-1365.

28 Dournes G, Grodzki D, Macey J, et al. Quiet Submillimeter MR imaging of the lung is feasible with a PETRA sequence at 1.5 T. Radiology 2015; 276: 258-265.

29 Gruden JF. CT in Idiopathic pulmonary fibrosis: diagnosis and beyond. AJR Am J Roentgenol 2016; 206 : $495-507$.

30 Kauczor HU, Hanke A, Van Beek EJ. Assessment of lung ventilation by MR imaging: current status and future perspectives. Eur Radiol 2002; 12: 1962-1970.

31 Lee CU, White DB, Sykes AM. Establishing a chest MRI practice and its clinical applications: our insight and protocols. J Clin Imaging Sci 2014; 4: 17.

32 Molinari F, Eichinger M, Risse F, et al. Navigator-triggered oxygen-enhanced MRI with simultaneous cardiac and respiratory synchronization for the assessment of interstitial lung disease. J Magn Reson Imaging 2007; 26: 1523-1529.

33 Fain SB, Korosec FR, Holmes JH, et al. Functional lung imaging using hyperpolarized gas MRI. J Magn Reson Imaging 2007; 25: 910-923.

34 Lederlin M, Bauman G, Eichinger M, et al. Functional MRI using Fourier decomposition of lung signal: reproducibility of ventilation- and perfusion-weighted imaging in healthy volunteers. Eur J Radiol 2013; 82: $1015-1022$

35 Ohno Y, Chen Q, Hatabu H. Oxygen-enhanced magnetic resonance ventilation imaging of lung. Eur J Radiol 2001; 37: 164-171.

36 Ohno Y, Hatabu H, Takenaka D, et al. Dynamic oxygen-enhanced MRI reflects diffusing capacity of the lung. Magn Reson Med 2002; 47: 1139-1144.

37 Triphan SM, Breuer FA, Gensler D, et al. Oxygen enhanced lung MRI by simultaneous measurement of T1 and T2 * during free breathing using ultrashort TE. J Magn Reson Imaging 2015; 41: 1708-1714

38 Stadler A, Stiebellehner L, Jakob PM, et al. Quantitative and $\mathrm{O}_{2}$ enhanced MRI of the pathologic lung: findings in emphysema, fibrosis, and cystic fibrosis. Int J Biomed Imaging 2007; 2007: 23624.

39 Stadler A, Jakob PM, Griswold M, et al. T1 mapping of the entire lung parenchyma: influence of the respiratory phase in healthy individuals. J Magn Reson Imaging 2005; 21: 759-764.

40 Stadler A, Jakob PM, Griswold M, et al. T1 mapping of the entire lung parenchyma: influence of respiratory phase and correlation to lung function test results in patients with diffuse lung disease. Magn Reson Med 2008; 59: 96-101.

41 Ohno Y, Nishio M, Koyama H, et al. Oxygen-enhanced MRI for patients with connective tissue diseases: comparison with thin-section CT of capability for pulmonary functional and disease severity assessment. Eur $J$ Radiol 2014; 83: 391-397.

42 Kruger SJ, Nagle SK, Couch MJ, et al. Functional imaging of the lungs with gas agents. J Magn Reson Imaging 2016; 43: 295-315.

43 Ebner L, Kammerman J, Driehuys B, et al. The role of hyperpolarized 129xenon in MR imaging of pulmonary function. Eur I Radiol 2017; 86: 343-352. 
44 Pusterla $\mathrm{O}$, Bauman G, Wielpütz $\mathrm{MO}$, et al. Rapid 3D in vivo $1 \mathrm{H}$ human lung respiratory imaging at $1.5 \mathrm{~T}$ using ultra-fast balanced steady-state free precession. Magn Reson Med 2017; 78: 1059-1069.

45 Prosch H, Schaefer-Prokop CM, Eisenhuber E, et al. CT protocols in interstitial lung diseases a survey among members of the European Society of Thoracic Imaging and a review of the literature. Eur Radiol 2013; 23: $1553-1563$.

46 Lavelle LP, Brady D, McEvoy S, et al. Pulmonary fibrosis: tissue characterization using late-enhanced MRI compared with unenhanced anatomic high-resolution CT. Diagn Interv Radiol 2017; 23: 106-111.

47 Mirsadraee S, Tse M, Kershaw L, et al. T1 characteristics of interstitial pulmonary fibrosis on 3T MRI - a predictor of early interstitial change. Quant Imaging Med Surg 2016; 6: 42-49.

48 King MA, Bergin CJ, Ghadishah E, et al. Detecting pulmonary abnormalities on magnetic resonance images in patients with usual interstitial pneumonitis: effect of varying window settings and gadopentetate dimeglumine. Acad Radiol 1996; 3: 300-307.

49 Kim ES, Keating GM. Pirfenidone: a review of its use in idiopathic pulmonary fibrosis. Drugs 2015; 75: 219-230.

50 El-Sherief AH, Gilman MD, Healey TT, et al. Clear vision through the haze: a practical approach to ground-glass opacity. Curr Probl Diagn Radiol 2014; 43: 140-158.

51 Lutterbey G, Grohé C, Gieseke J, et al. Initial experience with lung-MRI at 3.0T: comparison with CT and clinical data in the evaluation of interstitial lung disease activity. Eur J Radiol 2007; 61: 256-261.

52 Buzan MT, Eichinger M, Kreuter M, et al. T2 mapping of CT remodelling patterns in interstitial lung disease. Eur Radiol 2015; 25: 3167-3174.

53 Luna A, Sánchez-Gonzalez J, Caro P. Diffusion-weighted imaging of the chest. Magn Reson Imaging Clin N Am 2011; 19: 69-94.

54 Ciet P, Serra G, Andrinopoulou ER, et al. Diffusion weighted imaging in cystic fibrosis disease: beyond morphological imaging. Eur Radiol 2016; 26: 3830-3839.

55 Gaeta M, Blandino A, Scribano E, et al. Chronic infiltrative lung diseases: value of gadolinium-enhanced MRI in the evaluation of disease activity - early report. Chest 2000; 117: 1173-1178.

56 Sergiacomi G, Bolacchi F, Cadioli M, et al. Combined pulmonary fibrosis and emphysema: 3D time-resolved MR angiographic evaluation of pulmonary arterial mean transit time and time to peak enhancement. Radiology 2010; 254: 601-608.

57 Tsuchiya N, Yamashiro T, Murayama S. Decrease of pulmonary blood flow detected by phase contrast MRI is correlated with a decrease in lung volume and increase of lung fibrosis area determined by computed tomography in interstitial lung disease. Eur J Radiol 2016; 85: 1581-1585.

58 Suga $\mathrm{K}$, Tsukuda $\mathrm{T}$, Awaya $\mathrm{H}$, et al. Interactions of regional respiratory mechanics and pulmonary ventilatory impairment in pulmonary emphysema: assessment with dynamic MRI and xenon-133 single-photon emission CT. Chest 2000; 117: 1646-1655.

59 Iwasawa $\mathrm{T}$, Kagei S, Gotoh T, et al. Magnetic resonance analysis of abnormal diaphragmatic motion in patients with emphysema. Eur Respir J 2002; 19: 225-231.

60 Harlaar L, Ciet P, van der Ploeg AT, et al. Imaging of respiratory muscles in neuromuscular disease: a review. Neuromuscul Disord 2018; 28: 246-256.

61 Marinelli JP, Levin DL, Vassallo R, et al. Quantitative assessment of lung stiffness in patients with interstitial lung disease using MR elastography. J Magn Reson Imaging 2017; 46: 365-374.

62 Triphan SMF, Biederer J, Burmester K, et al. Design and application of an MR reference phantom for multicentre lung imaging trials. PLoS One 2018; 13: e0199148. 